\title{
Endobronchial coil therapy in severe emphysema: 6-month outcomes from a Swiss National Registry
}

\author{
Jean-Marc Fellrath ${ }^{1}$, Thomas Scherer ${ }^{2}$, Daniel P. Franzen ${ }^{3}$, Alban Lovis ${ }^{4}$, Christophe von Garnier $^{5}$, \\ Jérôme Plojoux ${ }^{6}$, Paola M. Soccal ${ }^{6,7}$
}

${ }^{1}$ Department of Pulmonary Medicine, Pourtales Hospital, Neuchatel, Switzerland; ${ }^{2}$ LungenZentrum Hirslanden, Zurich, Switzerland; ${ }^{3}$ Department of Pulmonology, University Hospital Zurich, Zurich, Switzerland; ${ }^{4}$ Pneumology Division, Centre Hospitalier Universitaire Vaudois, Lausanne, Switzerland; ${ }^{5}$ Respiratory Medicine Department, Inselspital, University of Bern, Bern, Switzerland; ${ }^{6}$ Division of Pulmonary Medicine, Geneva University Hospitals, Geneva, Switzerland; ${ }^{7}$ Faculty of Medicine, University of Geneva, Geneva, Switzerland

Contributions: (I) Conception and design: JM Fellrath, DP Franzen, PM Soccal; (II) Administrative support: All authors; (III) Provision of study materials or patients: All authors; (IV) Collection and assembly of data: All authors; (V) Data analysis and interpretation: All authors; (VI) Manuscript writing: All authors; (VII) Final approval of manuscript: All authors.

Correspondence to: Paola M. Soccal. Division of Pulmonary Medicine, Geneva University Hospitals 4, Rue Gabrielle-Perret-Gentil, CH-1211 Geneva 14, Switzerland. Email: Paola.Soccal@hcuge.ch.

Background: Endobronchial coils have been demonstrated in three randomized, controlled trials to improve quality of life, exercise tolerance, and lung function in patients with severe emphysema. This therapy is CE-marked and commercially available in Switzerland. Coil treated patients are followed in a post-market Swiss registry to collect safety and effectiveness data in routine clinical practice.

Methods: The Swiss coil registry was initiated in October 2013. At the end of November 2016, an interim analysis of all 64 patients treated in five centers was performed to evaluate safety and effectiveness at six months post treatment.

Results: patients had completed bilateral treatment with 6-month follow up at the time of data analysis. Patients had very severe, symptomatic emphysema and hyperinflation [38\% male, mean age 66 years, BMI 24, FEV1 30\% pred., residual volume (RV) 247\% pred., 6-minute walking distance (6-MWD) $272 \mathrm{~m}$, St. George Respiratory Questionnaire (SGRQ) 57 points]. Up to 6 months following treatment, seven serious adverse events (SAE) were reported in 6/29 patients. No device removals were necessary. At 6 months, responder rates [\% achieving the minimal clinically important difference (MCID)] were as follows: RV (-0.35 L) 76\%; FEV1 (+10\%) 57\%; SGRQ (-4 points) 87\%; 6MWD (+26 m) 60\%.

Conclusions: Endobronchial coil therapy performed in expert centers in Switzerland yields high 6-month responder rates across all relevant outcome

Keywords: Chronic obstructive pulmonary disease (COPD); emphysema; bronchoscopic lung volume reduction; coils; registry

Submitted Mar 28, 2018. Accepted for publication Apr 09, 2018.

doi: $10.21037 /$ jtd.2018.04.53

View this article at: http://dx.doi.org/10.21037/jtd.2018.04.53

\section{Introduction}

The emphysematous phenotype of Chronic obstructive pulmonary disease (COPD), with smoking as the main etiology has an estimated global prevalence of $1.8 \%$ (1).

The pathogenic mechanism of emphysema is destruction of alveolar walls, irreversible airway obstruction, loss of elastic recoil, air trapping, and thus a reduced gas exchange area. Clinical prognosis and quality of life in end-stage emphysema is very poor, with concomitant diseases increasing the risk of potentially life-threatening complications. Symptoms in emphysema-dominant patients 
are associated with dyspnea driven by lung hyperinflation (static and dynamic), and reduction of hyperinflation by reducing lung volume in the most diseased area of the lung is the objective of therapeutic intervention (2).

Optimal medical management of emphysema includes smoking cessation, pulmonary rehabilitation and physical activity training, supplemental oxygen as required, and pharmacotherapy. Nevertheless, symptoms such as dyspnea and impaired quality of life may become overwhelming and, thus, invasive treatment strategies, such as lung transplantation and lung volume reduction (LVR) are indicated. Only a minority of patients are suitable for lung transplantation due to shortage of donor organs, comorbidities and advanced age (in Switzerland 54 COPD patients underwent lung transplantation between 1993 and 2007) (3). Therefore, LVR is a promising treatment option in emphysema patients with hyperinflation. Lung volume reduction by surgery (LVRS) has been shown to improve lung function, 6MWD and quality of life in a subset of patients (2). However, postoperative morbidity and mortality remain concerns for this frail population, and LVRS has not been shown to benefit patients with homogenous disease. Several bronchoscopic lung volume reduction (BLVR) procedures have been intensively investigated in randomized trials. BLVR using endobronchial coils and valves are included in the GOLD2017 guidelines and are routinely applied throughout Europe (4).

The PneumRx ${ }^{\circledR}$ Endobronchial Coils have been demonstrated in three randomized, controlled trials to improve quality of life, exercise tolerance, and lung function in bilaterally treated patients (5-7). The coils are shapememory nitinol implants bronchoscopically deployed into the areas of lung parenchyma most damaged by emphysema $(8,9)$. Coils improve lung function by reducing lung volume and tethering airways, restoring airway patency and reducing airway collapse during exhalation and exercise $(5,10)$. Because the coil acts by a simple mechanical action and is not a blocking device, these effects are achieved immediately irrespective of collateral ventilation. This device is deployed via a simple catheter-based delivery system through a fiber-optic bronchoscope and requires no incision.

\section{Methods}

The PneumRx European registry (NCT01806636) is a prospective post market multi-center registry of patients treated in routine clinical practice throughout Europe.
Inclusion and exclusion criteria are in accordance with the company's Instructions for Use (IFU) and indications for coil therapy. Registry participants are patients scheduled to undergo coil treatment who consent to have their clinical data collected in the Registry. All patients had provided informed consent before undergoing treatment. Protocols and consent forms were approved by the local Ethics committees (Lead EC: Commission cantonale d'éthique de la recherche CCER, Geneva, ID: GE 14-017) prior to study enrollment. Data were de-identified and captured on web-based electronic Case Report Forms (eCRFs). The study is conducted under EEC MDD, EN ISO 14155:2011 and all applicable local regulations.

In Switzerland, coil therapy is used in 6 expert centers (Inselspital Bern, L'hôpital Neuchâtelois-Pourtalès, Hôpitaux Universitaires de Genève, Lungenzentrum Hirslanden, University Hospital Zurich, Centre Hospitalier Universitaire Vaudois). All treating physicians are boardcertified pulmonologists with interventional experience, and all received procedural training from the company. Participating sites have facilities that support the practice of interventional bronchoscopy and are able to perform comprehensive patient screening and selection as well as postoperative patient management. Patients had severe emphysema, were on optimal medical therapy, and ideally had completed pulmonary rehabilitation and smoking cessation prior to consenting to treatment. Patients underwent high resolution CT scanning to assess emphysema distribution and exclude suspicious findings, such as nodules suspicious for lung cancer, bronchiectasis, and severe bullous disease.

\section{Study population}

Adult patients diagnosed with homogeneous or heterogeneous emphysema who were appropriate for coil treatment based on the CE-Mark Approved IFU and who have been scheduled for coil treatment were considered candidates for the registry. Patients had residual volume $(\mathrm{RV})$ of at least $175 \%$ predicted, post bronchodilator forced expiratory volume in 1 second (FEV1) of $\leq 45 \%$ predicted and a normal international normalized ratio (INR) in the absence of anticoagulation therapy. Patients were not eligible for the coil treatment if they had known uncontrolled pulmonary hypertension defined by systolic $\mathrm{RV}$ pressure $>50 \mathrm{mmHg}$, giant bullae $>1 / 3$ lung volume, and were on antithrombotic treatment (except for aspirin) which could not be stopped 7 days prior to treatment. All 
patients who were treated with coils since October 2013 were enrolled in the Swiss registry.

\section{Devices and procedures}

Endobronchial coils are available in 3 lengths: 100, 125, and $150 \mathrm{~mm}$. The coil delivery system consists of a cartridge to house the straightened coil for deployment, a catheter for deployment, a guidewire and a specialized forcep for release or retrieval of the coil. The deployment procedure has been previously described (10). The peri-procedural patient management (e.g., prophylactic treatment, control chest X-ray(s) or CT(s), or post procedural control bronchoscopies) is left to the discretion of the treating physician to adhere to local standards for patient management following bronchoscopic interventions, and was not captured in the registry.

\section{Follow-up}

Per the Registry protocol, post-procedure patient followup was based on the individual institution's standard of care. Registry specific follow-up visits were recommended at 6 months and 12 months, and then annually up to 3 years counted from the date of the first procedure. The baseline visit included medical examination, pulmonary function tests (body plethysmography, spirometry), 6-minute walk test (6MWT) on room air or carrying oxygen when needed, and medication history. Quality of life (QOL) was assessed by the St. George`s Respiratory Questionnaire (SGRQ) and dyspnea by the modified Medical Research Council (mMRC) 5-level dyspnoea scale. All baseline, procedure, and follow-up data were collected via eCRFs and entered into the electronic data capture (EDC) database. Adverse events were recorded at each study visit.

\section{Study endpoints}

The primary endpoint of this study was the change in quality of life from baseline to post treatment followup as assessed by the SGRQ (total points). Secondary endpoints included changes in lung function measurements [(RV) (L), RV/total lung capacity (TLC) (\%), FEV1 (L), FEV1/forced vital capacity (FVC) (\%)] and changes in exercise capacity assessed by the $6 \mathrm{MWT}$ between baseline and post treatment follow-up.

Responder rates were evaluated using the minimal clinical important differences (MCIDs) that have been reported for FEV1, L +10\% (11), RV -0.35 L (12), 6MWD +26 m (13), and SGRQ -4 points total score (14).

Safety endpoints included occurrence of serious adverse events (SAE) including any device malfunction or failures.

\section{Statistical analyses}

Patient demographics, baseline characteristics, procedure characteristics, device usage and hospital stay are summarized using descriptive statistics. Serious Adverse events (SAEs) are summarized by number and percent of subjects as well as number of events for the Treatment Recovery Period (30 Days following either procedure), 31-91, 92-182, and through 6 months of follow-up. Effectiveness data is summarized using descriptive statistics. Results at 6-month follow-up were compared to baseline values and analyzed using paired t-test for continuous variables (alpha level 0.05). No adjustments were made for multiple comparisons.

\section{Results}

Twenty-nine patients had completed bilateral treatment and had completed a 6-month follow-up visit between October 2013 and November 2016 (Figure 1). Of these, an additional two patients died (both prior to the 6-month follow-up visit).

Baseline and treatment characteristics of the patients in this analysis are shown in Table 1. Sixty-nine percent of patients had heterogeneous emphysema as determined by investigators and $31 \%$ had homogeneous emphysema. These patients had very advanced emphysema with severe hyperinflation, with mean FEV1 30\% pred., and RV 247\% pred. They were functionally limited with very poor quality of life, with mean 6MWD of $272 \mathrm{~m}$, and mean SGRQ score of 57 points. Pulmonary rehabilitation status was documented in 14/29 (48\%) patients, of whom $9(64 \%)$ had completed pulmonary rehabilitation. Patients received mean 23 coils in two separate procedures and completed the 6-month post treatment follow-up visit at median 204 days (range, 124 to 272 days). The median hospital stay after each coil procedure was 1.0 day; mean procedure time was 47 minutes which included 15 minutes under fluoroscopy.

At 6 months, patients reported considerable improvement in their quality of life following bilateral coil treatment. SGRQ decreased by $-13.9 \pm 11.9$ points compared to baseline $(\mathrm{P}<0.0001)$ (Table 2), with an $87 \%$ SGRQ responder rate. $61 \%$ of patients achieved twice the 


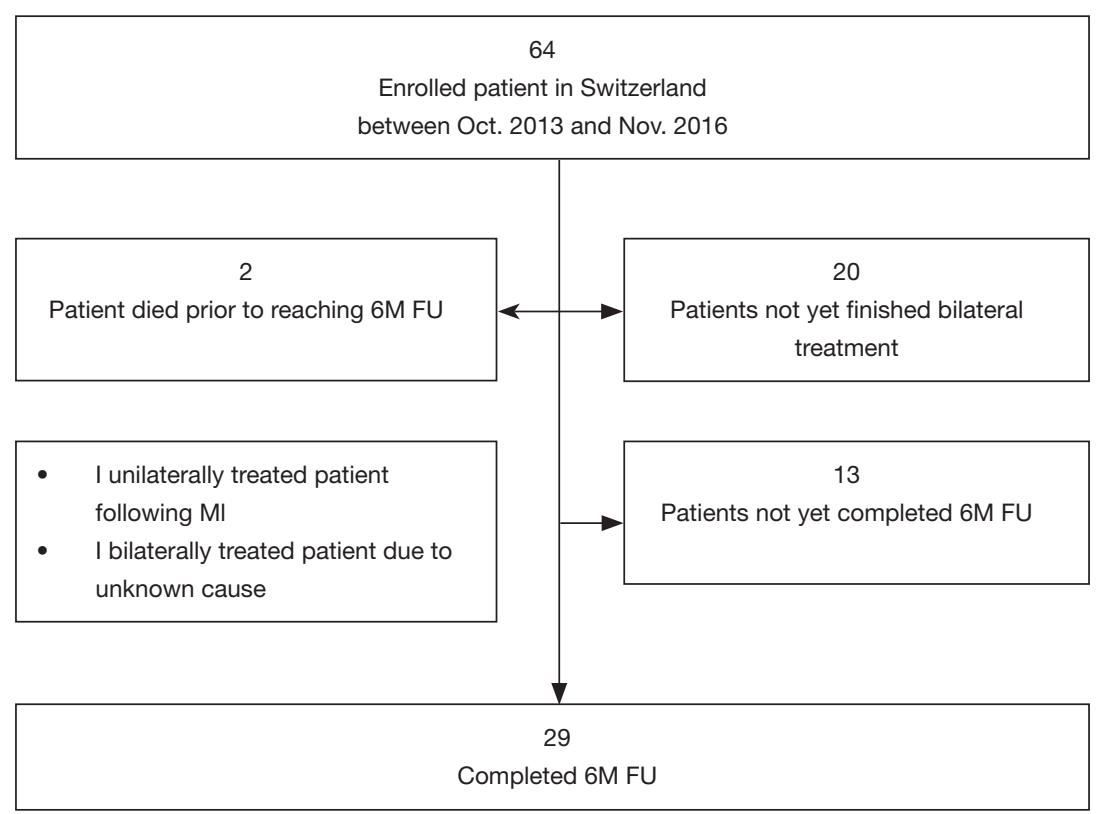

Figure 1 Patient accountability. FU, follow-up.

MCID in SGRQ response ( $\geq 8$ points decrease) (Table 3).

Patients demonstrated significant increases in $6 \mathrm{MWD}$, with mean increase of $53.4 \pm 66.9 \mathrm{~m}(\mathrm{P}=0.0021)$ (Table 2). A $6 \mathrm{MWD}$ responder rate of $60 \%$ was observed at 6 -month follow-up (Table 3).

$\mathrm{FEV}_{1}$ increased by $0.14 \pm 0.22 \mathrm{~L}$ at 6 months $(\mathrm{P}=0.0021)$, with $57 \%$ of patients achieving MID level response at $10 \%$ threshold. RV decreased by $-0.91 \pm 1.04 \mathrm{~L}$ at 6 months compared to baseline ( $\mathrm{P}=0.0002) ; 76 \%$ of patients achieved the MID of $-0.35 \mathrm{~L}$. The RV/TLC [\%] also showed a statistically significant decrease at 6 months after treatment (mean change $-7.72 \pm 11.19 ; \mathrm{P}=0.0021$ ).

A total of 7 SAEs were reported in 6 of 29 bilaterally treated patients (Table 4) through 6 months; 5 SAEs occurred within the first 30 days of either treatment [1 pneumonia, 2 pneumothorax, 1 acute myocardial infarction (MI), 1 chest pain]. Two patients developed pneumonia after 30 days but within the 6-month followup period. All SAEs were resolved. Among the total Swiss registry population of 64 patients, two deaths were reported within the 6-month follow-up (1 myocardial infraction, one unconfirmed cause of death); neither was deemed procedure or device related. No device removals were necessary.

\section{Discussion}

We present our experience with endobronchial coil treatment as part of routine clinical practice in Switzerland. The key limitation of this analysis is the small population of patients with completed 6-month follow-up; however, real-world effectiveness as well as real-world challenges of patient follow-up are important to integration of novel therapeutic approaches, and should be described. Because there was no control arm, a placebo effect cannot be ruled out, nor can responder bias. However, the RENEW trial reported outcomes in coil treated patients $v s$. a control arm, with statistically significant between-group differences in 6MWD, FEV1, SGRQ, and RV/TLC favoring the coil treatment group at one-year post treatment, and a highly clinically significant between-group difference in SGRQ of -8.9 points $(\mathrm{P}<0.001)$ at 1 -year post treatment $(7)$. Because not all centers offered alternate endoscopic lung volume reduction treatment options, a strength of this study is avoidance of selection bias, whereby patients with severe hyperinflation and highly heterogenous disease (presumably the optimal "responder profile") might be directed toward other therapies while patients with homogenous disease, who typically demonstrate lower magnitude of benefit, might preferentially be directed toward coils. Our physiologic outcomes in patients treated with endobronchial coils are in fact comparable to 6-month published outcomes in the post-market setting for a larger cohort treated with endobronchial valves (15).

A strength of this analysis is that all patients treated 
Table 1 Baseline characteristics (all patients, $\mathrm{N}=29$ )

\begin{tabular}{|c|c|c|c|c|}
\hline Baseline parameters & $\mathrm{n}$ & Mean $\pm \mathrm{SD} / \mathrm{n}(\%)$ & Median & Range \\
\hline \multicolumn{5}{|l|}{ Demographics } \\
\hline Age, years & 29 & $65.76 \pm 9.27$ & 66 & $37-83$ \\
\hline $\mathrm{BMI}, \mathrm{kg} / \mathrm{m}^{2}$ & 29 & $23.66 \pm 4.94$ & 23 & $16.16-35.56$ \\
\hline Gender: female/male & 29 & $18(62.07) / 11(37.93)$ & & \\
\hline Emphysema : heterogeneous/homogeneous & 29 & $20(68.97) / 9(31.03)$ & & \\
\hline 6MWD (m) & 23 & $272.17 \pm 88.03$ & 270 & $107-482$ \\
\hline SGRQ (points) & 29 & $56.58 \pm 10.36$ & 57 & $36-76$ \\
\hline mMRC (points) & 29 & $2.66 \pm 0.77$ & 2 & \\
\hline RV/TLC (\%) & 28 & $68.78 \pm 9.02$ & 69 & $45-85$ \\
\hline FEV1 (L) & 28 & $0.72 \pm 0.20$ & 1 & $0.4-1.1$ \\
\hline FEV1 \% predicted & 28 & $29.67 \pm 7.78$ & 29 & $17-47$ \\
\hline FEV1/FVC (\%) & 28 & $36.84 \pm 9.49$ & 36 & $22-59$ \\
\hline Prior rehabilitation: yes/no & 14 & $9(64.29) / 5(35.71)$ & & \\
\hline \multicolumn{5}{|l|}{ Procedures } \\
\hline Days between treatments (Tx): $T x 1$ to $T x 2$ & 29 & $85.45 \pm 50.35$ & 82 & $21-210$ \\
\hline Total coils & 29 & $23.24 \pm 5.10$ & 22 & $18-45$ \\
\hline Fluoroscopy time (min) post Tx1 & 28 & $14.75 \pm 11.93$ & 10 & $5-45$ \\
\hline Fluoroscopy time (min) post Tx2 & 28 & $15.93 \pm 14.96$ & 10 & $5-50$ \\
\hline Hospital days post Tx1 & 29 & $1.79 \pm 1.08$ & 1 & $1-5$ \\
\hline Hospital days post Tx2 & 29 & $2.41 \pm 2.69$ & 1 & $1-12$ \\
\hline
\end{tabular}

6MWD, 6-minute walk distance; BMI, body mass index; FEV1, forced expiratory volume in 1 second; FVC, forced vital capacity, mMRC, modified medical research council; RV, residual volume; SGRQ, St. George Respiratory Questionnaire; TX, treatment.

with coils in Switzerland were included in the registry and were thus considered in this analysis. Another strength is that patients in this multi-center registry achieved a high response rate across all 4 endpoints of interest in COPD, despite a very broad range of baseline characteristics of our patients compared to previously published trials. Our median age was 66 but the range is 37 to 83 years, our median 6MWD is $270 \mathrm{~m}$ with a range of 107 to $482 \mathrm{~m}$, our median RV\% pred. is $247 \%$ with a range of $147 \%$ to $392 \%$, and our FEV1\% pred. is $29 \%$ with a range of $17-47 \%$. We believe our data suggest that careful and holistic patient selection, taking into account the patient's medical (including psychosocial) history, exacerbation history, stability of other comorbidities, and commitment 
Table 2 Efficacy results: change from baseline at 6-month FU (all patients, N=29)

\begin{tabular}{lccc}
\hline Change from baseline at 6-month FU & $\mathrm{n}$ & Mean \pm SD & $\mathrm{P}$ for all groups \\
\hline 6MWD (m) & 20 & $53.40 \pm 66.92$ & 0.0021 \\
SGRQ (points) & 23 & $-13.88 \pm 11.89$ & $<0.0001$ \\
FEV1 (L) & 28 & $0.14 \pm 0.22$ & 0.0021 \\
FEV1/FVC (\%) & 28 & $-0.46 \pm 6.01$ & 0.6909 \\
RV (L) & 25 & $-0.91 \pm 1.04$ & 0.0002 \\
RV/TLC (\%) & 25 & $-7.72 \pm 11.19$ & 0.0021 \\
\hline
\end{tabular}

6MWD, 6-minute walk distance; FEV1, forced expiratory volume in 1 second; FU, follow-up; FVC, forced vital capacity, RV, residual volume; SGRQ, St. George Respiratory Questionnaire; TLC, total lung capacity.

Table 3 Efficacy results: responder rates (MCID) at 6-month FU for bilaterally treated patients (all patients)

\begin{tabular}{lcc}
\hline MCID responder rates at 6-month FU & $n / N$ & Percent \\
\hline 6MWD responder ( $\geq 26$ m) & $12 / 20$ & 60.0 \\
SGRQ responder ( $\geq 4$ points) & $20 / 23$ & 87.0 \\
SGRQ responder ( $\geq 8$ points) & $14 / 23$ & 60.9 \\
RV responder ( $\geq 0.35$ L) & $19 / 25$ & 76.0 \\
FEV1 responder ( $\geq 10 \%)$ & $16 / 28$ & 57.0 \\
Two or more MCIDs responder^ & $21 / 29$ & 72.4 \\
\hline
\end{tabular}

$\wedge$, at least two MCID responses among 6MWD, SGRQ (4 points), FEV1 and RV; missing values have been counted as non-response. $6 \mathrm{MWD}$, 6-minute walk distance; FEV1, forced expiratory volume in 1 second; FU, follow-up; MCID, minimal clinically important difference; RV, residual volume; SGRQ, St. George Respiratory Questionnaire.

Table 4 SAEs of interest (all patients $\mathrm{N}=29$ )

\begin{tabular}{|c|c|c|c|c|}
\hline SAEs of interest & $\begin{array}{c}\text { Subjects, } 0-30 \mathrm{~d} \text { post } \\
\mathrm{Tx} 1, \mathrm{n}(\%)\end{array}$ & $\begin{array}{c}\text { Events, } 0-30 \mathrm{~d} \text { post } \\
\operatorname{Tx} 1(\mathrm{n})\end{array}$ & $\begin{array}{l}\text { Through } 6 \text { months subjects } \\
(0-182 \text { d post } T \times 1) n(\%)\end{array}$ & $\begin{array}{c}\text { Through } 6 \text { months events } \\
\text { (0-182 d post Tx1), n }\end{array}$ \\
\hline Total & $5(17.24)$ & 5 & $6(20.69)$ & 7 \\
\hline Chest pain & $1(3.45)$ & 1 & $1(3.45)$ & 1 \\
\hline Pneumonia & $1(3.45)$ & 1 & $3(10.34)$ & 3 \\
\hline
\end{tabular}

SAEs, serious adverse events; TX, treatment.

to self-care may be a stronger predictor of good outcomes than any particular baseline factor. Due to the complexity and variability of the severe emphysema patient profile, we also deemed notable that $72 \%$ of our patients achieved at least 2 MCID level responses among the 4 outcomes of interest (SGRQ, 6MWD, FEV1 and RV) (Table 3). Since endobronchial trials tend to demonstrate high SGRQ improvements, we consider a "2 or more MCID" threshold as potentially indicative of overall "responsiveness", where either a functional or physiologic endpoint supports the patient's reported improvement in quality of life.

Our data represents the first analysis of coil therapy implemented in centers of excellence throughout Switzerland. We believe that some learnings can be 
derived from these data. As previously described, a factor influencing responsiveness to lung volume reduction is heterogeneity of emphysema $(2,16)$. Patients with homogenous disease are generally not candidates for lung volume reduction surgery, endobronchial valves, or other non-implant therapies under clinical investigation (i.e., steam or glue). However, in the RENEW randomized coil trial, $77 \%$ of patients had homogeneous emphysema based on visual assessment (7). In our registry, $67 \%$ of patients have heterogeneous emphysema, supporting the notion that heterogeneity may predict larger magnitudes of improvement from any intervention, and that therapeutic success must be considered from the perspective of the patient (i.e., quality of life improvement) and the limitations of the disease profile (i.e., other comorbidities or degree of heterogeneity).

In Switzerland, single photon emission computed tomography (SPECT) perfusion is part of the standard diagnostic work up for patients who are evaluated for lung volume reduction procedures. Because LVRS and lobar blocking therapies "disconnect" the treated/resected area from ventilation, perfusion status plays a critical role in determining optimal lobe targets. The situation is less clear for coil treatment, which is not a blocking therapy, and hence treated lobes remain ventilated. In Switzerland, patients were treated based on visual assessment of the emphysema, combined with semi-quantitative SPECT perfusion analysis. Patients usually received treatment in the lobes with the lowest perfusion, which is typically associated with areas of higher emphysematous destruction; however, this was left to the discretion of the treating physician. SPECT data were not captured in the registry and quantitative CT (qCT) analyses were not performed. Thus, it remains speculative if SPECT and qCT to determine lobe targeting for coils might have contributed to the very good effectiveness data in this study.

\section{Conclusions}

Endobronchial coil therapy in Switzerland is safe and yields very high 6-month responder rates across all relevant outcome measures. Our findings suggest that endobronchial coil therapy is an excellent therapeutic option for carefully selected patients when offered in experienced interventional centers.

\section{Acknowledgements}

PneumRx provided statistical support and writing support for the development of this manuscript.

\section{Footnote}

Conflicts of Interest: The authors have no conflicts of interest to declare.

Ethical Statement: All patients had provided informed consent before undergoing treatment. Protocols and consent forms were approved by the local Ethics committees (Lead EC: Commission cantonale d'éthique de la recherche CCER, Geneva, ID: GE 14-017) prior to study enrollment.

\section{References}

1. Raherison C, Girodet PO. Epidemiology of COPD. European Respiratory Review 2009;18:213-21.

2. Fishman A, Martinez F, Naunheim K, et al. A randomized trial comparing lung-volume-reduction surgery with medical therapy for severe emphysema. $\mathrm{N}$ Engl J Med 2003;348:2059-73.

3. Lahzami S, Bridevaux PO, Soccal PM, et al. Survival impact of lung transplantation for COPD. Eur Respir J 2010;36:74-80.

4. Global Initiative for Chronic Obstructive Lung Disease (GOLD): Global strategy for the diagnosis, management, and prevention of chronic obstructive pulmonary disease, 2017 report. Available online: http://goldcopd.org/gold2017-global-strategy-diagnosis-management-preventioncopd/

5. Shah PL, Zoumot Z, Singh S, et al. Endobronchial coils for the treatment of severe emphysema with hyperinflation (RESET): a randomised controlled trial. Lancet Respir Med 2013;1:233-40.

6. Deslee G, Mal H, Dutau H, et al. Lung volume reduction coil treatment vs usual care in patients with severe emphysema: The REVOLENS Randomized Clinical Trial. JAMA 2016;315:175-84.

7. Sciurba FC, Criner GJ, Strange C, et al. Effect of endobronchial coils vs usual care on exercise tolerance in patients with severe emphysema: the RENEW Randomized Clinical Trial. JAMA 2016;315:2178-89.

8. Vijayan VK. Chronic obstructive pulmonary disease. Indian J Med Res 2013;137:251-69.

9. McDonough JE, Yuan R, Suzuki M, et al. Small-airway obstruction and emphysema in chronic obstructive pulmonary disease. N Engl J Med 2011;365:1567-75.

10. Klooster K, Ten Hacken NH, Franz I, et al. Lung volume 
reduction coil treatment in chronic obstructive pulmonary disease patients with homogeneous emphysema: a prospective feasibility trial. Respiration 2014;88:116-25.

11. Donohue JF. Minimal clinically important differences in COPD lung function. COPD 2005;2:111-24.

12. Hartman JE, Ten Hacken NH, Klooster K, et al. The minimal important difference for residual volume in patients with severe emphysema. Eur Respir J 2012;40:1137-41.

13. Puhan MA, Chandra D, Mosenifar Z, et al. The minimal important difference of exercise tests in severe COPD. Eur
Respir J 2011;37:784-90.

14. Jones PW. St. George's Respiratory Questionnaire: MCID. COPD 2005;2:75-9.

15. Skowasch D, Fertl A, Schwick B, et al. A Long-Term Follow-Up Investigation of Endobronchial Valves in Emphysema (the LIVE Study): Study Protocol and SixMonth Interim Analysis Results of a Prospective Five-Year Observational Study. Respiration 2016;92:118-26.

16. Sciurba FC, Ernst A, Herth FJ, et al. A randomized study of endobronchial valves for advanced emphysema. N Engl J Med 2010;363:1233-44.

Cite this article as: Fellrath JM, Scherer T, Franzen DP, Lovis A, von Garnier C, Plojoux J, Soccal PM. Endobronchial coil therapy in severe emphysema: 6-month outcomes from a Swiss National Registry. J Thorac Dis 2018;10(Suppl 23):S2711-S2718. doi: $10.21037 /$ jtd.2018.04.53 